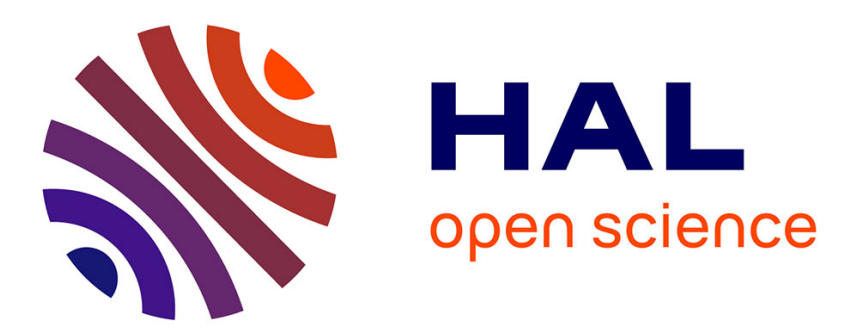

\title{
Uniform controllability of semidiscrete approximations of parabolic control systems
}

Stéphane Labbé, Emmanuel Trélat

\section{To cite this version:}

Stéphane Labbé, Emmanuel Trélat. Uniform controllability of semidiscrete approximations of parabolic control systems. Systems and Control Letters, 2006, 55 (7), pp.597-609. hal-00086374

\section{HAL Id: hal-00086374 \\ https://hal.science/hal-00086374}

Submitted on 18 Jul 2006

HAL is a multi-disciplinary open access archive for the deposit and dissemination of scientific research documents, whether they are published or not. The documents may come from teaching and research institutions in France or abroad, or from public or private research centers.
L'archive ouverte pluridisciplinaire HAL, est destinée au dépôt et à la diffusion de documents scientifiques de niveau recherche, publiés ou non, émanant des établissements d'enseignement et de recherche français ou étrangers, des laboratoires publics ou privés. 


\title{
Uniform controllability of semidiscrete approximations of parabolic control systems
}

\author{
Stéphane Labbé, Emmanuel Trélat* \\ Université Paris-Sud, Math., AN-EDP, UMR 8628, Bât. 425, 91405 Orsay cedex, France.
}

\begin{abstract}
Controlling an approximation model of a controllable infinite dimensional linear control system does not necessarily yield a good approximation of the control needed for the continuous model. In the present paper, under the main assumptions that the discretized semigroup is uniformly analytic, and that the control operator is mildly unbounded, we prove that the semidiscrete approximation models are uniformly controllable. Moreover, we provide a computationally efficient way to compute the approximation controls. An example of application is implemented for the one- and two-dimensional heat equation with Neumann boundary control.
\end{abstract}

Keywords: Controllability, partial differential equation, discretization, observability inequality, Hilbert uniqueness method.

\section{Introduction}

Consider an infinite dimensional linear control system

$$
\dot{y}(t)=A y(t)+B u(t), \quad y(0)=y_{0},
$$

where the state $y(t)$ belongs to a Hilbert space $X$, the control $u(t)$ belongs to a Hilbert space $U$, $A: D(A) \rightarrow X$ is an operator, and $B$ is a control operator (in general, unbounded) on $U$. Discretizing this partial differential equation, using for instance a finite difference, or a finite element

\footnotetext{
*Corresponding author. Tel.: +33-169156673; fax: +33-1-69156718. E-mail addresses: Stephane.Labbe@math.u-psud.fr (S. Labbé), Emmanuel.Trelat@math.u-psud.fr (E. Trélat).
}

scheme, leads to a family of finite dimensional linear control systems

$$
\dot{y}_{h}(t)=A_{h} y_{h}(t)+B_{h} u_{h}(t), \quad y_{h}(0)=y_{0 h},
$$

where $y_{h}(t) \in X_{h}$ and $u_{h}(t) \in U_{h}$, for $0<h<h_{0}$.

Let $y_{1} \in X$; if the control system (1) is controllable in time $T$, then there exists a solution $y(\cdot)$ of (1), associated with a control $u$, such that $y(T)=y_{1}$. The following question arises naturally: is it possible to find controls $u_{h}$, for $0<h<h_{0}$, converging to the control $u$ as the mesh size $h$ of the discretization process tends to zero, and such that the associated trajectories $y_{h}(\cdot)$, solutions of $(2)$, converge to $y(\cdot)$ ? Moreover, does there exist an efficient algorithmic way to determine the controls $u_{h}$ ?

For controllable linear control systems of the type (1), we have available many methods in order to realize the controllability. A well known method, adapted to numerical implementations, is the Hilbert Uniqueness Method (HUM), introduced in [14], which consists in minimizing a cost function, namely, the $L^{2}$ norm of the control. In this paper, we investigate the above question in the case where controllability of (1) is achieved using the HUM method. Our objective is to establish conditions ensuring a uniform controllability property of the family of discretized control systems (2), and to establish a computationally feasible approximation method for realizing controllability.

The question of uniform controllability and/or observability of the family of approximation control systems (2) has been investigated by E. Zuazua and collaborators in a series of articles $[4,8,13,16,18,26,30,31,32,33,35]$, for different discretization processes, on different exam- 
ples. When the observability constant of the finite dimensional approximation systems does not depend on $h$, one says that the property of uniform observability holds. For classical finite difference schemes, a uniform boundary observability property holds for one-dimensional heat equations [16], beam equations [13], Schrödinger equations [33], but does not hold for 1-D wave equations [8]. It is actually well known that discretization processes generate high frequency spurious solutions that do not exist in the continuous problem, and that may lead, in the exact controllability problem, to the divergence of the control approximations.

In the parabolic case, the strong dissipative properties induced by the analyticity of the semigroup totally damp out these spurious high frequencies in 1-D (see [16]), but do not suffice however in general in the multi-dimensional case (see [33]). In the hyperbolic case, where there is no such strong damping, the divergence phenomenon of controls may be drastic (see $[8,33,35]$ ).

The discretization framework used in this paper is in the same spirit as the one of $[1,2,6$, $9,12,15,20]$. In these references, approximation results are provided for the linear quadratic regulator (LQR) problem in the parabolic case, that show, in particular, the convergence of the controls of the semidiscrete models to the control of the continuous model. However, in the LQR problem, the final point is not fixed, and the exact controllability problem is a very different matter.

In the present paper, we prove a uniform controllability property of the discretized models (2), in the case where the operator $A$ generates an analytic semigroup. Of course, due to regularization properties, the control system (1) is not exactly controllable in general. Hence, we focus on exact null controllability. Our main result, Theorem 3.1 , states that, for an exactly null controllable parabolic system (1), under standard assumptions on the discretization process (that are satisfied for most of classical schemes), if the discretized semigroup is uniformly analytic (see [12]), and if the degree of unboundedness of the control operator $B$ with respect to $A$ (see [21]) is lower than $1 / 2$, then the approximating control systems are uniformly controllable, in the following sense: we are able to construct a bounded sequence of approximat- ing controls $u_{h}$, that converge to the HUM control of the continuous model; morever, the norm of $y_{h}(T)$, where $y_{h}(\cdot)$ is the corresponding solution of (2), tends to zero as $h$ tends to zero, with a rate of convergence that is estimated. A uniform observability and admissibility type inequality is proved. We stress that we do not prove a uniform exact null controllability property for the approximating systems (2). A minimization procedure to compute the approximation controls is provided, and an important fact in view of the numerical implementation is that the uniform property implies a uniform conditioning of the gradient method. Note that the condition on the degree of unboundedness of $B$ is satisfied for distributed controls (that is, if $B$ is bounded), and, if $B$ is unbounded, it is for instance satisfied for the heat equation with Neumann boundary control, but not with Dirichlet boundary control.

The outline of the article is as follows. In Section 2, we briefly review some well known facts on controllability for finite and infinite dimensional linear control systems. The main result is stated in Section 3, and proved in Section 4. An example of application, and numerical simulations, are provided in Section 5, for the one- and twodimensional heat equation with Neumann boundary control. In Section 6, we formulate some further comments, and open problems. Section 7 is an appendix devoted to the proof of a lemma.

\section{A short review on control- lability}

\subsection{Controllability of finite dimen- sional linear control systems}

Let $T>0$ fixed. Consider the linear control system

$$
\dot{x}(t)=A x(t)+B u(t),
$$

where $x(t) \in \mathbb{R}^{n}, A$ is a $(n \times n)$-matrix, $B$ is a $(n \times m)$-matrix, with real coefficients, and $u(\cdot) \in$ $L^{2}\left(0, T ; \mathbb{R}^{m}\right)$.

Let $x_{0} \in \mathbb{R}^{n}$. The system (3) is controllable from $x_{0}$ in time $T$ if, for every $x_{1} \in \mathbb{R}^{n}$, there exists $u(\cdot) \in L^{2}\left(0, T ; \mathbb{R}^{m}\right)$ so that the corresponding solution $x(\cdot)$ of $(3)$, with $x(0)=x_{0}$, satisfies 
$x(T)=x_{1}$.

It is well known that the system (3) is controllable in time $T$ if and only if the matrix $\int_{0}^{T} \mathrm{e}^{(T-t) A} B B^{*} \mathrm{e}^{(T-t) A^{*}} d t$, called Gramian of the system, is nonsingular (here, $M^{*}$ denotes the transpose of the matrix $M$ ). In finite dimension, this is equivalent to the existence of $\alpha>0$ so that

$$
\int_{0}^{T}\left\|B^{*} \mathrm{e}^{(T-t) A^{*}} \psi\right\|^{2} d t \geq \alpha\|\psi\|^{2},
$$

for every $\psi \in \mathbb{R}^{n}$ (observability inequality).

It is also well known that, if such a linear system is controllable from $x_{0}$ in time $T>0$, then it is controllable in any time $T^{\prime}$, and from any initial state $x_{0}^{\prime} \in \mathbb{R}^{n}$. Indeed, another necessary and sufficient condition for controllability is the Kalman condition $\operatorname{rank}\left(B, A B, \ldots, A^{n-1} B\right)=n$, which is independent on $x_{0}$ and $T$.

\subsection{Controllability of linear partial differential equations in Hilbert spaces}

In this section, we review some known facts on controllability of infinite dimensional linear control systems in Hilbert spaces (see [28, 29]).

Throughout the paper, the notation $L(E, F)$ stands for the set of linear continuous mappings from $E$ to $F$, where $E$ and $F$ are Hilbert spaces.

Let $X$ be a Hilbert space. Denote by $\langle,\rangle_{X}$ the inner product on $X$, and by \|\|$_{X}$ the associated norm. Let $S(t)$ denote a strongly continuous semigroup on $X$, of generator $(A, D(A))$. Let $X_{-1}$ denote the completion of $X$ for the norm $\|x\|_{-1}=\left\|(\beta I-A)^{-1} x\right\|$, where $\beta \in \rho(A)$ is fixed. Note that $X_{-1}$ does not depend on the specific value of $\beta \in \rho(A)$. The space $X_{-1}$ is isomorphic to $\left(D\left(A^{*}\right)\right)^{\prime}$, the dual space of $D\left(A^{*}\right)$ with respect to the pivot space $X$, and $X \subset X_{-1}$, with a continuous and dense embedding. The semigroup $S(t)$ extends to a semigroup on $X_{-1}$, still denoted $S(t)$, whose generator is an extension of the operator $A$, still denoted $A$. With these notations, $A$ is a linear operator from $X$ to $X_{-1}$.

Let $U$ be a Hilbert space. Denote by $\langle,\rangle_{U}$ the inner product on $U$, and by \|\|$_{U}$ the associated norm.
A linear continuous operator $B: U \rightarrow X_{-1}$ is admissible for the semigroup $S(t)$ if every solution of

$$
\dot{y}(t)=A y(t)+B u(t),
$$

with $y(0)=y_{0} \in X$ and $u(\cdot) \in L^{2}(0,+\infty ; U)$, satisfies $y(t) \in X$, for every $t \geq 0$. In this case, $y(\cdot) \in H^{1}(0,+\infty ; X)$, the differential equation (5) on $X_{-1}$ holds almost everywhere on $[0,+\infty)$, and

$$
y(t)=S(t) y_{0}+\int_{0}^{t} S(t-s) B u(s) d s
$$

for every $t \geq 0$.

For $T>0$, define $L_{T}: L^{2}(0, T ; U) \rightarrow X_{-1}$ by

$$
L_{T} u=\int_{0}^{T} S(T-s) B u(s) d s .
$$

A control operator $B \in \mathcal{L}\left(U, X_{-1}\right)$ is admissible if and only if $\operatorname{Im} L_{T} \subset X$, for some (and hence for every) $T>0$. Note that the adjoint $L_{T}^{*}$ of $L_{T}$ satisfies

$$
L_{T}^{*} y_{0}(t)=B^{*} S(T-t)^{*} y_{0} \text { a.e. on }[0, T],
$$

for every $y_{0} \in D\left(A^{*}\right)$.

Let $B \in \mathcal{L}\left(U, X_{-1}\right)$ denote an admissible control operator.

For $y_{0} \in X$, and $T>0$, the system (5) is exactly controllable from $y_{0}$ in time $T$ if, for every $y_{1} \in X$, there exists $u(\cdot) \in L^{2}(0, T ; U)$ so that the corresponding solution of (5), with $y(0)=y_{0}$, satisfies $y(T)=y_{1}$.

It is clear that the system (5) is exactly controllable from $y_{0}$ in time $T$ if and only if $L_{T}$ is onto, that is $\operatorname{Im} L_{T}=X$. In particular, if the system (5) is exactly controllable from $y_{0}$ in time $T$, then it is exactly controllable from any point $y_{0}^{\prime} \in X$ in time $T$. One says that the system (5) is exactly controllable in time $T$. It is well known that the system (1) is exactly controllable in time $T$ if and only if there exists $\alpha>0$ so that

$$
\int_{0}^{T}\left\|B^{*} S^{*}(t) \psi\right\|_{U}^{2} d t \geq \alpha\|\psi\|_{X}^{2},
$$

for every $\psi \in D\left(A^{*}\right)$ (observability inequality).

For $T>0$, the system (5) is said to be exactly null controllable in time $T$ if, for every $y_{0} \in X$, 
there exists $u(\cdot) \in L^{2}(0, T ; U)$ so that the corresponding solution of $(5)$, with $y(0)=y_{0}$, satisfies $y(T)=0$. The system (1) is exactly null controllable in time $T$ if and only if there exists $\alpha>0$ so that

$$
\int_{0}^{T}\left\|B^{*} S^{*}(t) \psi\right\|_{U}^{2} d t \geq \alpha\left\|S(T)^{*} \psi\right\|_{X}^{2},
$$

for every $\psi \in D\left(A^{*}\right)$.

Remark 2.1. Assume that $B$ is admissible and that the control system (5) is exactly null controllable in time $T$. Let $y_{0} \in X$. For every $\psi \in D\left(A^{*}\right)$, set

$$
J(\psi)=\frac{1}{2} \int_{0}^{T}\left\|B^{*} S(t)^{*} \psi\right\|_{U}^{2} d t+\left\langle S(T)^{*} \psi, y_{0}\right\rangle_{X} .
$$

The functional $J$ is strictly convex, and, from the observability inequality (10), is coercive. Hence, it admits a unique minimizer $\varphi \in D\left(A^{*}\right)$. Define the control $u$ by

$$
u(t)=B^{*} S(T-t)^{*} \varphi
$$

for every $t \in[0, T]$, and let $y(\cdot)$ be the solution of $(5)$, such that $y(0)=y_{0}$, associated with the control $u$. Then, one has $y(T)=0$, and moreover, $u$ is the control of minimal $L^{2}$ norm, among all controls whose associated trajectory satisfies $y(T)=0$.

This remark proves that observability implies controllability, and gives a constructive way to build the control of minimal $L^{2}$ norm (see [32]). This is more or less the contents of the Hilbert Uniqueness Method (see [14]). Hence, in what follows, we refer to the control (12) as the HUM control.

\section{The main result}

Let $X$ and $U$ be Hilbert spaces, and let $A$ : $D(A) \rightarrow X$ be a linear operator, generating a strongly continuous semigroup $S(t)$ on $X$. Let $B \in L\left(U, D\left(A^{*}\right)^{\prime}\right)$ be a control operator. We make the following assumptions.

$\left(\mathbf{H}_{\mathbf{1}}\right)$ The semigroup $S(t)$ is analytic.
Therefore (see [19]), there exist positive real numbers $C_{1}$ and $\omega$ such that

$\|S(t) y\|_{X} \leq C_{1} \mathrm{e}^{\omega t}\|y\|_{X},\|A S(t) y\|_{X} \leq C_{1} \frac{\mathrm{e}^{\omega t}}{t}\|y\|_{X}$,

for all $t>0$ and $y \in D(A)$, and such that, if we set $\hat{A}=A-\omega I$, then the fractional powers $(-\hat{A})^{\theta}$ of $\hat{A}$ are well defined, for $\theta \in[0,1]$, and there holds

$$
\left\|(-\hat{A})^{\theta} S(t) y\right\|_{X} \leq C_{1} \frac{\mathrm{e}^{\omega t}}{t^{\theta}}\|y\|_{X}
$$

for all $t>0$ and $y \in D(A)$.

Of course, inequalities (13) hold as well if one replaces $A$ by $A^{*}, S(t)$ by $S(t)^{*}$, for $y \in D\left(A^{*}\right)$.

Moreover, if $\rho(A)$ denotes the resolvent set of $A$, then there exists $\delta \in(0, \pi / 2)$ such that

$$
\rho(A) \supset \Delta_{\delta}=\left\{\omega+\rho \mathrm{e}^{i \theta}|\rho>0,| \theta \mid \leq \frac{\pi}{2}+\delta\right\} .
$$

For $\lambda \in \rho(A)$, denote by $R(\lambda, A)=(\lambda I-A)^{-1}$ the resolvent of $A$. It follows from the previous estimates that there exists $C_{2}>0$ such that

$$
\|R(\lambda, A)\|_{L(X)} \leq \frac{C_{2}}{|\lambda-\omega|},\|A R(\lambda, A)\|_{L(X)} \leq C_{2},
$$

for every $\lambda \in \Delta_{\delta}$, and

$$
\|R(\lambda, \hat{A})\|_{L(X)} \leq \frac{C_{2}}{|\lambda|},\|\hat{A} R(\lambda, \hat{A})\|_{L(X)} \leq C_{2},
$$

for every $\lambda \in\left\{\Delta_{\delta}+\omega\right\}$. Similarly, inequalities (16) and (17) hold as well with $A^{*}$ and $\hat{A}^{*}$.

$\left(\mathbf{H}_{2}\right)$ The degree of unboundedness of $B$ is lower than $1 / 2$, i.e., there exists $\gamma \in[0,1 / 2)$ such that

$$
B \in L\left(U, D\left(\left(-\hat{A}^{*}\right)^{\gamma}\right)^{\prime}\right) .
$$

In these conditions, the domain of $B^{*}$ is $D\left(B^{*}\right)=$ $D\left(\left(-\hat{A}^{*}\right)^{\gamma}\right)$, and there exists $C_{3}>0$ such that

$$
\left\|B^{*} \psi\right\|_{U} \leq C_{3}\left\|\left(-\hat{A}^{*}\right)^{\gamma} \psi\right\|_{X}
$$

for every $\psi \in D\left(\left(-\hat{A}^{*}\right)^{\gamma}\right)$.

Note that this assumption implies that the control operator $B$ is admissible.

We next introduce adapted approximation assumptions, inspired by [12] (see also [1, 2, 6, 9, $15,20])$. Consider two families $\left(X_{h}\right)_{0<h<h_{0}}$ and $\left(U_{h}\right)_{0<h<h_{0}}$ of finite dimensional spaces, where $h$ is the discretization parameter. 
$\left(\mathbf{H}_{\mathbf{3}}\right)$ For every $h \in\left(0, h_{0}\right)$, there exist linear mappings $P_{h}: D\left(\left(-\hat{A}^{*}\right)^{1 / 2}\right)^{\prime} \rightarrow X_{h}$ and $\widetilde{P}_{h}:$ $X_{h} \rightarrow D\left(\left(-\hat{A}^{*}\right)^{1 / 2}\right)$ (resp., there exist linear mappings $Q_{h}: U \rightarrow U_{h}$ and $\left.\widetilde{Q}_{h}: U_{h} \rightarrow U\right)$, satisfying the following requirements:

$\left(\mathbf{H}_{3.1}\right)$ For every $h \in\left(0, h_{0}\right)$, there holds

$$
P_{h} \widetilde{P}_{h}=i d_{X_{h}} \text {, and } Q_{h} \widetilde{Q}_{h}=i d_{U_{h}} .
$$

$\left(\mathbf{H}_{3.2}\right)$ There exist $s>0$ and $C_{4}>0$ such that there holds, for every $h \in\left(0, h_{0}\right)$,

$$
\begin{gathered}
\left\|\left(I-\widetilde{P}_{h} P_{h}\right) \psi\right\|_{X} \leq C_{4} h^{s}\left\|A^{*} \psi\right\|_{X}, \\
\left\|\left(-\hat{A}^{*}\right)^{\gamma}\left(I-\widetilde{P}_{h} P_{h}\right) \psi\right\|_{X} \\
\leq C_{4} h^{s(1-\gamma)}\left\|A^{*} \psi\right\|_{X}
\end{gathered}
$$

for every $\psi \in D\left(A^{*}\right)$, and

$$
\left\|\left(I-\widetilde{Q}_{h} Q_{h}\right) u\right\|_{U} \underset{h \rightarrow 0}{\longrightarrow} 0,
$$

for every $u \in U$, and

$$
\left\|\left(I-\widetilde{Q}_{h} Q_{h}\right) B^{*} \psi\right\|_{U} \leq C_{4} h^{s(1-\gamma)}\left\|A^{*} \psi\right\|_{X}
$$

for every $\psi \in D\left(A^{*}\right)$.

Note that (22) makes sense since, by assumption, $\gamma<1 / 2$, and thus, $\operatorname{Im} \widetilde{P}_{h} \subset$ $D\left(\left(-\hat{A}^{*}\right)^{1 / 2}\right) \subset D\left(\left(-\hat{A}^{*}\right)^{\gamma}\right)$.

For every $h \in\left(0, h_{0}\right)$, the vector space $X_{h}$ (resp. $\left.U_{h}\right)$ is endowed with the norm \|\|$_{X_{h}}$ (resp., \|\|$_{U_{h}}$ ) defined by

$$
\left\|y_{h}\right\|_{X_{h}}=\left\|\widetilde{P}_{h} y_{h}\right\|_{X}
$$

for $y_{h} \in X_{h}$ (resp., $\left\|u_{h}\right\|_{U_{h}}=\left\|\widetilde{Q}_{h} u_{h}\right\|_{U}$, for $\left.u_{h} \in U_{h}\right)$. In these conditions, it is clear that

$$
\left\|\widetilde{P}_{h}\right\|_{L\left(X_{h}, X\right)}=\left\|\widetilde{Q}_{h}\right\|_{L\left(U_{h}, U\right)}=1,
$$

for every $h \in\left(0, h_{0}\right)$. Moreover, it follows from (21), (22), (23), and from the BanachSteinhaus Theorem, that there exists $C_{5}>0$ such that

$$
\left\|P_{h}\right\|_{L\left(X, X_{h}\right)} \leq C_{5} \text {, and }\left\|Q_{h}\right\|_{L\left(U, U_{h}\right)} \leq C_{5},
$$

and

$$
\left\|\left(-\hat{A}^{*}\right)^{\gamma}\left(I-\widetilde{P}_{h} P_{h}\right) \psi\right\|_{X} \leq C_{5}\left\|\left(-\hat{A}^{*}\right)^{\gamma} \psi\right\|_{X},
$$

for all $h \in\left(0, h_{0}\right)$ and $\psi \in D\left(\left(-\hat{A}^{*}\right)^{\gamma}\right)$.
$\left(\mathbf{H}_{\mathbf{3 . 3}}\right)$ For every $h \in\left(0, h_{0}\right)$, there holds

$$
P_{h}=\widetilde{P}_{h}^{*}, \text { and } Q_{h}=\widetilde{Q}_{h}^{*},
$$

where the adjoint operators are considered with respect to the pivot spaces $X$, $U, X_{h}$, and $U_{h}$.

Note that this assumption indeed holds for most of classical schemes (Galerkin or spectral approximations, centered finite differences, ...).

$\left(\mathbf{H}_{3.4}\right)$ There exists $C_{6}>0$ such that

$$
\left\|B^{*} \widetilde{P}_{h} \psi_{h}\right\|_{U} \leq C_{6} h^{-\gamma s}\left\|\psi_{h}\right\|_{X_{h}},
$$

for all $h \in\left(0, h_{0}\right)$ and $\psi_{h} \in X_{h}$.

For every $h \in\left(0, h_{0}\right)$, we define the approximation operators $A_{h}^{*}: X_{h} \rightarrow X_{h}$ of $A^{*}$, and $B_{h}^{*}: X_{h} \rightarrow U_{h}$ of $B^{*}$, by

$$
A_{h}^{*}=P_{h} A^{*} \widetilde{P}_{h} \text {, and } B_{h}^{*}=Q_{h} B^{*} \widetilde{P}_{h} .
$$

Due to $\left(H_{3.3}\right)$, it is clear that $B_{h}=P_{h} B \widetilde{Q}_{h}$, for every $h \in\left(0, h_{0}\right)$. On the other hand, we set $A_{h}=$ $\left(A_{h}^{*}\right)^{*}$ (with respect to the pivot space $X_{h}$ ). Note that, if $A$ is selfadjoint, then $A_{h}=P_{h} A \widetilde{P}_{h}$.

$\left(\mathbf{H}_{4}\right)$ The following properties hold:

$\left(\mathbf{H}_{4.1}\right)$ The family of operators $\mathrm{e}^{t A_{h}}$ is uniformly analytic, in the sense that there exists $C_{7}>0$ such that

$$
\begin{aligned}
& \left\|\mathrm{e}^{t A_{h}}\right\|_{L\left(X_{h}\right)} \leq C_{7} \mathrm{e}^{\omega t}, \\
& \left\|A_{h} \mathrm{e}^{t A_{h}}\right\|_{L\left(X_{h}\right)} \leq C_{7} \frac{\mathrm{e}^{\omega t}}{t},
\end{aligned}
$$

for all $t>0$ and $h \in\left(0, h_{0}\right)$.

$\operatorname{Under}\left(H_{4.1}\right)$, there exists $C_{8}>0$ such that

$$
\left\|R\left(\lambda, A_{h}\right)\right\|_{L\left(X_{h}\right)} \leq \frac{C_{8}}{|\lambda-\omega|},
$$

for every $\lambda \in \Delta_{\delta}$. Note that (32) and (33) hold as well if one replaces $A_{h}$ with $A_{h}^{*}$. 
$\left(\mathbf{H}_{4.2}\right)$ There exists $C_{9}>0$ such that, for every $f \in X$ and every $h \in\left(0, h_{0}\right)$, the respective solutions of $\hat{A}^{*} \psi=f$ and $\hat{A}_{h}^{*} \psi_{h}=P_{h} f$ satisfy

$$
\left\|P_{h} \psi-\psi_{h}\right\|_{X_{h}} \leq C_{9} h^{s}\|f\|_{X} .
$$

In other words, there holds $\| P_{h} \hat{A}^{*-1}-$ $\hat{A}_{h}^{*-1} P_{h} \|_{L\left(X, X_{h}\right)} \leq C_{9} h^{s}$. This is a (strong) rate of convergence assumption.

Remark 3.1. Assumptions $\left(H_{3}\right)$ and $\left(H_{4.2}\right)$ hold for most of the classical numerical approximation schemes, such as Galerkin methods, spectral methods, centered finite difference schemes, ... As noted in [12], the assumption $\left(H_{4.1}\right)$ of uniform analyticity is not standard, and has to be checked in each specific case. However, it can be shown to hold, under Assumption $\left(H_{1}\right)$, provided the bilinear form associated with $A_{h}$ is uniformly coercive (see [3] for the selfadjoint case, and [11, Lemma 4.2] for the general nonselfadjoint case).

The main result of the paper is the following.

Theorem 3.1. Under the previous assumptions, the control system $\dot{y}=A y+B u$ is exactly null controllable in time $T>0$, if and only if the family of discretized control systems $\dot{y}_{h}=A_{h} y_{h}+B_{h} u_{h}$ is uniformly controllable in the following sense. There exist $\beta>0, h_{1}>0$, and positive real numbers $c, c^{\prime}$, such that the uniform observability and admissibility inequality

$$
\begin{gathered}
c\left\|\mathrm{e}^{T A_{h}^{*}} \psi_{h}\right\|_{X_{h}}^{2} \leq \int_{0}^{T}\left\|B_{h}^{*} \mathrm{e}^{t A_{h}^{*}} \psi_{h}\right\|_{U_{h}}^{2} d t+h^{\beta}\left\|\psi_{h}\right\|_{X_{h}}^{2} \\
\leq c^{\prime}\left\|\psi_{h}\right\|_{X_{h}}^{2}
\end{gathered}
$$

holds, for every $h \in\left(0, h_{1}\right)$ and every $\psi_{h} \in X_{h}$.

In these conditions, for every $y_{0} \in X$, and every $h \in\left(0, h_{1}\right)$, there exists a unique $\varphi_{h} \in X_{h}$ minimizing the functional

$$
\begin{gathered}
J_{h}\left(\psi_{h}\right)=\frac{1}{2} \int_{0}^{T}\left\|B_{h}^{*} \mathrm{e}^{t A_{h}^{*}} \psi_{h}\right\|_{U_{h}}^{2} d t+\frac{1}{2} h^{\beta}\left\|\psi_{h}\right\|_{X_{h}}^{2} \\
+\left\langle\mathrm{e}^{T A_{h}^{*}} \psi_{h}, P_{h} y_{0}\right\rangle_{X_{h}}
\end{gathered}
$$

and the sequence $\left(\widetilde{Q}_{h} u_{h}\right)_{0<h<h_{1}}$, where the control $u_{h}$ is defined by

$$
u_{h}(t)=B_{h}^{*} \mathrm{e}^{(T-t) A_{h}^{*}} \varphi_{h},
$$

for every $t \in[0, T]$, converges weakly (up to a subsequence), in the space $L^{2}(0, T ; U)$, to a control $u$ such that the solution of

$$
\dot{y}=A y+B u, y(0)=y_{0},
$$

satisfies $y(T)=0$. For every $h \in\left(0, h_{1}\right)$, let $y_{h}(\cdot)$ denote the solution of

$$
\dot{y}_{h}=A_{h} y_{h}+B_{h} u_{h}, y_{h}(0)=P_{h} y_{0} .
$$

Then,

- $y_{h}(T)=-h^{\beta} \varphi_{h}$;

- for every $t \in[0, T]$, the sequence $\left(\widetilde{P}_{h} y_{h}(t)\right)_{0<h<h_{1}} \quad$ converges strongly (up to a subsequence), in the space $X$, to $y(t)$;

- the sequence $\left(\widetilde{P}_{h} y_{h}(\cdot)\right)_{0<h<h_{1}}$ converges strongly (up to a subsequence), in the space $L^{2}(0, T ; X)$, to $y(\cdot)$.

Furthermore, there exists $M>0$ such that

$$
\int_{0}^{T}\|u(t)\|_{U}^{2} d t \leq M^{2}\left\|y_{0}\right\|_{X}^{2},
$$

and, for every $h \in\left(0, h_{1}\right)$,

$$
\begin{aligned}
\int_{0}^{T}\left\|u_{h}(t)\right\|_{U_{h}}^{2} d t & \leq M^{2}\left\|y_{0}\right\|_{X}^{2}, \\
h^{\beta}\left\|\varphi_{h}\right\|_{X_{h}}^{2} & \leq M^{2}\left\|y_{0}\right\|_{X}^{2}, \\
\left\|y_{h}(T)\right\|_{X_{h}} & \leq M h^{\beta / 2}\left\|y_{0}\right\|_{X} .
\end{aligned}
$$

Remark 3.2. The left-hand side of (35) is a uniform observability type inequality for the control systems $\dot{y}_{h}=A_{h} y_{h}+B_{h} u_{h}$. The right-hand side of that inequality implies that the control operators $B_{h}$ are uniformly admissible.

Remark 3.3. From the numerical point of view, in order to construct approximating controls $u_{h}$, the procedure consists in minimizing the functional $J_{h}$ defined by (36), for instance using a gradient like method. Note the very important fact that the uniform inequality (35) implies a uniform conditioning of the minimization problem, and thus, the number of iterations needed in the gradient method is uniformly bounded with respect to $h$. This crucial property ensures the effective applicability of the algorithm. 
Note that the notion of uniform controllability provided by this result is relevant from the numerical point of view. Indeed, numerically, it is impossible to realize exactly zero.

Remark 3.4. As noticed in the introduction, we stress that this is not a result of uniform exact null controllability for the approximating systems (39). Such a result is indeed wrong in general: for two-dimensional heat equations, finite difference approximations are not uniformly exactly null controllable in general (see [33, 35]).

Furthermore, notice the following fact. On the one hand, exact null controllability of the continuous model implies approximate controllability, with the additional fact that controls remain bounded as $\varepsilon$ tends to zero (see [34]). On the other hand, if the continuous model is approximately controllable, and if the numerical scheme is convergent, then it is possible to prove, without using an assumption of analyticity of the semigroup, a property of uniform approximate controllability (see [32]). Combining these two facts leads to a similar result as the one of Theorem 3.1, not requiring the assumption of analyticity. However, it is not possible a priori with this argument to derive a uniform observability type inequality, and hence, to ensure that the number of iterations of the gradient method is uniformly bounded. In [32], it is indeed explained, in the case of the wave equation, that such a uniform observability type inequality cannot hold, even though one adds a term in $O\left(h^{\beta}\right)$ with respect to the norm of $X_{h}$. In this particular case, a stronger norm is actually necessary. This remark indicates that, in our analysis and method, the analyticity assumption cannot be removed.

Remark 3.5. A similar result holds if the control system $\dot{y}=A y+B u$ is exactly controllable in time $T>0$. However, due to Assumption $\left(H_{1}\right)$, the semigroup $S(t)$ enjoys in general regularity properties. Therefore, the solution $y(\cdot)$ of the control system may belong to a subspace of $X$, whatever the control $u$ is. For instance, in the case of the heat equation with a Dirichlet or Neumann boundary control, the solution is a smooth function of the state variable $x$, as soon as $t>0$, for every control and initial condition $y_{0} \in L^{2}$. Hence, exact controllability does not hold in this case in $L^{2}$.
The theorem states that the controls $u_{h}$ defined by (37) tend to a control $u$ realizing the exact null controllability for (38). One may wonder under which assumptions the control $u$ is the HUM control such that $y(T)=0$ (see Remark 2.1). The following result provides an answer.

Proposition 3.2. With the notations of Theorem 3.1, if the sequence of real numbers $\left\|\varphi_{h}\right\|_{X_{h}}$, $0<h<h_{1}$, is moreover bounded, then the control $u$ is the unique HUM control such that $y(T)=0$. Moreover, the sequence $\left(\widetilde{Q}_{h} u_{h}\right)_{0<h<h_{1}}$ converges strongly (up to a subsequence), in the space $L^{2}(0, T ; U)$, to the control $u$.

A sufficient condition on $y_{0} \in X$, ensuring the boundedness of the sequence $\left(\left\|\varphi_{h}\right\|_{X_{h}}\right)_{0<h<h_{1}}$, is the following: there exists $\eta>0$ such that the control system $\dot{y}=A y+B u$ is exactly null controllable in time $t$, for every $t \in[T-\eta, T+\eta]$, and the trajectory $t \mapsto S(t) y_{0}$ in $X$, for $t \in[T-\eta, T+\eta]$, is not contained in a hyperplane of $X$.

An example where this situation indeed occurs is the following. Additionally to the previous assumptions, assume that the operator $A$ admits a Hilbertian basis of eigenvectors $e_{k}$, associated with eigenvalues $\lambda_{k}$, for $k \in \mathbb{N}$, satisfying

$$
\sum_{k=1}^{+\infty} \frac{-1}{\lambda_{k}}<+\infty \text {. }
$$

Let $y_{0}=\sum_{k \in \mathbb{N}} y_{0 k} e_{k}$ a point of $X$ such that $y_{0 k} \neq 0$, for every $k \in \mathbb{N}$. Then, the assumption of Proposition 3.2 is satisfied. Indeed, if the trajectory $t \mapsto S(t) y_{0}$ in $X$, for $t \in[T-\eta, T+$ $\eta]$, were contained in a hyperplane of $X$, there would exist $\Phi=\sum_{k \in \mathbb{N}} \Phi_{k} e_{k} \in X \backslash\{0\}$ so that $\sum_{k \in \mathbb{N}} \mathrm{e}^{\lambda_{k} t} y_{0 k} \Phi_{k}=0$, for every $t \in[T-\eta, T+\eta]$. It is well known that the condition (42) implies that the functions $\mathrm{e}^{\lambda_{k} t}, k \in \mathbb{N}$, are independent in $L^{2}$. Hence, $y_{0 k} \Phi_{k}=0$, for every $k \in \mathbb{N}$. This yields a contradiction.

\section{Proof of the main results}

\subsection{Proof of Theorem 3.1}

The proof is based on the following approximation lemma, whose proof readily follows that of $[12$, Lemma 4.3.1 p. 446]. To be self-contained, a proof of this lemma is provided in Appendix. 
Lemma 4.1. There exists $C_{10}>0$ such that, for all $t \in(0, T]$ and $h \in\left(0, h_{0}\right)$, there holds

$$
\begin{gathered}
\left\|\left(\mathrm{e}^{t A_{h}^{*}} P_{h}-P_{h} S(t)^{*}\right) \psi\right\|_{X_{h}} \leq C_{10} \frac{h^{s}}{t}\|\psi\|_{X}, \\
\left\|\widetilde{Q}_{h} B_{h}^{*} \mathrm{e}^{t A_{h}^{*}} \psi_{h}\right\|_{U} \leq \frac{C_{10}}{t^{\gamma}}\left\|\psi_{h}\right\|_{X_{h}},
\end{gathered}
$$

for all $\psi \in D\left(A^{*}\right)$ and $\psi_{h} \in X_{h}$, and moreover, for every $\theta \in[0,1]$,

$$
\begin{aligned}
& \left\|\widetilde{Q}_{h} B_{h}^{*} \mathrm{e}^{t A_{h}^{*}} \psi_{h}-B^{*} S(t)^{*} \widetilde{P}_{h} \psi_{h}\right\|_{U} \\
& \quad \leq C_{10} \frac{h^{s(1-\gamma) \theta}}{t^{\theta+(1-\theta) \gamma}}\left\|\psi_{h}\right\|_{X_{h}}, \forall \psi_{h} \in X_{h} .
\end{aligned}
$$

Remark 4.1. It follows from the proof of this lemma that (43) can be improved to

$$
\left\|\left(\mathrm{e}^{t A_{h}^{*}} P_{h}-P_{h} S(t)^{*}\right) \psi\right\|_{X_{h}} \leq C_{10} \frac{h^{s \theta}}{t^{\theta}}\|\psi\|_{X},
$$

for every $\psi \in D\left(A^{*}\right)$.

Let us prove that, if the system $\dot{y}=A y+B u$ is exactly null controllable, then the uniform inequality (35) holds. Since the degree of unboundedness $\gamma$ of the control operator $B$ is lower than $1 / 2$, there exists $\theta \in(0,1)$ such that $0<\theta+(1-$ $\theta) \gamma<1 / 2$.

For all $h \in\left(0, h_{0}\right)$ and $\psi_{h} \in X_{h}$, we have

$$
\begin{gathered}
\int_{0}^{T}\left\|\widetilde{Q}_{h} B_{h}^{*} \mathrm{e}^{t A_{h}^{*}} \psi_{h}\right\|_{U}^{2} d t=\int_{0}^{T}\left\|B^{*} S(t)^{*} \widetilde{P}_{h} \psi_{h}\right\|_{U}^{2} d t \\
+\int_{0}^{T}\left(\left\|\widetilde{Q}_{h} B_{h}^{*} \mathrm{e}^{t A_{h}^{*}} \psi_{h}\right\|_{U}^{2}-\left\|B^{*} S(t)^{*} \widetilde{P}_{h} \psi_{h}\right\|_{U}^{2}\right) d t .
\end{gathered}
$$

Since the control system $\dot{y}=A y+B u$ is exactly null controllable in time $T$, there exists a positive real number $c>0$ such that

$$
\int_{0}^{T}\left\|B^{*} S(t)^{*} \widetilde{P}_{h} \psi_{h}\right\|_{U}^{2} d t \geq c\left\|S(T)^{*} \widetilde{P}_{h} \psi_{h}\right\|_{X}^{2} .
$$

Using (21), (26), (13), and the estimate (43),

$$
\begin{aligned}
& \left\|S(T)^{*} \widetilde{P}_{h} \psi_{h}-\widetilde{P}_{h} \mathrm{e}^{T A_{h}^{*}} \psi_{h}\right\|_{X} \\
& \leq\left\|\left(I-\widetilde{P}_{h} P_{h}\right) S(T)^{*} \widetilde{P}_{h} \psi_{h}\right\|_{X} \\
& \quad+\left\|\widetilde{P}_{h}\left(P_{h} S(T)^{*} \widetilde{P}_{h} \psi_{h}-\mathrm{e}^{T A_{h}^{*}} \psi_{h}\right)\right\|_{X} \\
& \leq C_{4} h^{s}\left\|A^{*} S(T)^{*} \widetilde{P}_{h} \psi_{h}\right\|_{X} \\
& \quad+\left\|P_{h} S(T)^{*} \widetilde{P}_{h} \psi_{h}-\mathrm{e}^{T A_{h}^{*}} \psi_{h}\right\|_{X_{h}} \\
& \leq C_{11} h^{s}\left\|\psi_{h}\right\|_{X_{h}},
\end{aligned}
$$

where $C_{11}$ is a positive constant, independent on $h$. Hence, using (13) and (32), we get

$$
\begin{aligned}
& \left|\left\|S(T)^{*} \widetilde{P}_{h} \psi_{h}\right\|_{X}^{2}-\left\|\mathrm{e}^{T A_{h}^{*}} \psi_{h}\right\|_{X_{h}}^{2}\right| \\
& \leq\left\|S(T)^{*} \widetilde{P}_{h} \psi_{h}-\widetilde{P}_{h} \mathrm{e}^{T A_{h}^{*}} \psi_{h}\right\|_{X} \\
& \quad *\left(\left\|S(T)^{*} \widetilde{P}_{h} \psi_{h}\right\|_{X}+\left\|\mathrm{e}^{T A_{h}^{*}} \psi_{h}\right\|_{X_{h}}\right) \\
& \leq C_{12} h^{s}\left\|\psi_{h}\right\|_{X_{h}}^{2},
\end{aligned}
$$

where $C_{12}>0$ is independent on $h$. Therefore,

$$
\left\|S(T)^{*} \widetilde{P}_{h} \psi_{h}\right\|_{X}^{2} \geq\left\|\mathrm{e}^{T A_{h}^{*}} \psi_{h}\right\|_{X_{h}}^{2}-C_{12} h^{s}\left\|\psi_{h}\right\|_{X_{h}}^{2} .
$$

For the second term of the right-hand side of (47), one has, using (45), (44), (19) and (14),

$$
\begin{aligned}
& \left|\left\|\widetilde{Q}_{h} B_{h}^{*} \mathrm{e}^{t A_{h}^{*}} \psi_{h}\right\|_{U}^{2}-\left\|B^{*} S(t)^{*} \widetilde{P}_{h} \psi_{h}\right\|_{U}^{2}\right| \\
& \leq\left\|\widetilde{Q}_{h} B_{h}^{*} \mathrm{e}^{t A_{h}^{*}} \psi_{h}-B^{*} S(t)^{*} \widetilde{P}_{h} \psi_{h}\right\|_{U} \\
& \quad *\left(\left\|\widetilde{Q}_{h} B_{h}^{*} \mathrm{e}^{t A_{h}^{*}} \psi_{h}\right\|_{U}+\left\|B^{*} S(t)^{*} \widetilde{P}_{h} \psi_{h}\right\|_{U}\right) \\
& \leq C_{13} h^{s(1-\gamma) \theta} \frac{1}{t^{\theta+(1-\theta) \gamma+\gamma}}\left\|\psi_{h}\right\|_{X_{h}}^{2},
\end{aligned}
$$

where $C_{13}>0$ is independent on $h$. Since $\theta+(1-$ $\theta) \gamma+\gamma<1$, we get, by integration,

$$
\begin{aligned}
& \left|\int_{0}^{T}\left(\left\|\widetilde{Q}_{h} B_{h}^{*} \mathrm{e}^{t A_{h}^{*}} \psi_{h}\right\|_{U}^{2}-\left\|B^{*} S(t)^{*} \widetilde{P}_{h} \psi_{h}\right\|_{U}^{2}\right) d t\right| \\
& \quad \leq C_{14} h^{s(1-\gamma) \theta}\left\|\psi_{h}\right\|_{X_{h}}^{2} .
\end{aligned}
$$

If we choose a real number $\beta$ such that $0<\beta<$ $s(1-\gamma) \theta$, then the left-hand side of the inequality (35) (i.e., the uniform observability inequality) follows from (47), (48), (49), and (50), for $h \in\left(0, h_{1}\right)$, where $h_{1}>0$ is small enough. The right-hand side of (35), that is, the uniform admissibility inequality follows by integration of (44).

For $h \in\left(0, h_{1}\right)$, the functional $J_{h}$ is strictly convex, and, from (35), is coercive. Hence, it admits a unique minimum at $\varphi_{h} \in X_{h}$ so that

$$
0=\nabla J_{h}\left(\varphi_{h}\right)=M_{h}(T) \varphi_{h}+h^{\beta} \varphi_{h}+\mathrm{e}^{T A_{h}} P_{h} y_{0},
$$

where $M_{h}(T)=\int_{0}^{T} \mathrm{e}^{t A_{h}} B_{h} B_{h}^{*} \mathrm{e}^{t A_{h}^{*}} d t$ is the Gramian of the semidiscrete system. Then, the solution $y_{h}(\cdot)$ of $(39)$ satisfies

$$
\begin{aligned}
y_{h}(T) & =\mathrm{e}^{T A_{h}} y_{h}(0)+\int_{0}^{T} \mathrm{e}^{(T-t) A_{h}} B_{h} u_{h}(t) d t \\
& =\mathrm{e}^{T A_{h}} P_{h} y_{0}+M_{h}(T) \varphi_{h}=-h^{\beta} \varphi_{h} .
\end{aligned}
$$


Note that, since $J_{h}(0)=0$, there must hold, at the minimum, $J_{h}\left(\varphi_{h}\right) \leq 0$. Hence, using the observability inequality (35) and the Cauchy-Schwarz inequality, one gets

$$
\begin{gathered}
c\left\|\mathrm{e}^{T A_{h}^{*}} \varphi_{h}\right\|_{X_{h}}^{2} \leq \int_{0}^{T}\left\|B_{h}^{*} \mathrm{e}^{t A_{h}^{*}} \varphi_{h}\right\|_{U_{h}}^{2} d t+h^{\beta}\left\|\varphi_{h}\right\|_{X_{h}}^{2} \\
\leq 2\left\|\mathrm{e}^{T A_{h}^{*}} \varphi_{h}\right\|_{X_{h}}\left\|P_{h} y_{0}\right\|_{X_{h}},
\end{gathered}
$$

and thus,

$$
\left\|\mathrm{e}^{T A_{h}^{*}} \varphi_{h}\right\|_{X_{h}} \leq \frac{2}{c}\left\|P_{h} y_{0}\right\|_{X_{h}} .
$$

As a consequence, $\int_{0}^{T}\left\|B_{h}^{*} \mathrm{e}^{t A_{h}^{*}} \varphi_{h}\right\|_{U_{h}}^{2} d t \leq$ $\frac{4}{c}\left\|P_{h} y_{0}\right\|_{X_{h}}^{2}$, and $h^{\beta}\left\|\varphi_{h}\right\|_{X_{h}}^{2} \leq \frac{4}{c}\left\|P_{h} y_{0}\right\|_{X_{h}}^{2}$, and the estimates (41) follow.

In particular, the sequence $\left(\widetilde{Q}_{h} u_{h}\right)_{0<h<h_{1}}$ is bounded in $L^{2}(0, T ; U)$, and thus, up to a subsequence, converges to a control $u$. Let $y(\cdot)$ denote the solution of (38), associated with this control $u$. According to (7), one has

$$
\begin{aligned}
y(t) & =S(t) y_{0}+\int_{0}^{t} S(t-s) B u(s) d s \\
& =S(t) y_{0}+L_{t} u,
\end{aligned}
$$

for every $t \in[0, T]$, and, with a similar notation,

$$
\begin{aligned}
y_{h}(t) & =\mathrm{e}^{t A_{h}} P_{h} y_{0}+\int_{0}^{t} \mathrm{e}^{(t-s) A_{h}} B_{h} u_{h}(s) d s \\
& =\mathrm{e}^{t A_{h}} P_{h} y_{0}+L_{t h} u_{h},
\end{aligned}
$$

for every $h \in\left(0, h_{1}\right)$. We next derive the following lemma.

Lemma 4.2. For every $t \in[0, T]$, the sequence of operators $\widetilde{P}_{h} L_{t h} Q_{h}$ converges strongly to $L_{t}$ in the space $L\left(L^{2}(0, t ; U), X\right)$.

Proof. For every $v \in L^{2}(0, t ; U)$, using a dual version of (45), the Cauchy-Schwarz inequality, and the fact that $\theta+(1-\theta) \gamma<1 / 2$, one has

$$
\begin{aligned}
& \left\|\widetilde{P}_{h} L_{t h} Q_{h} v-L_{t} v\right\|_{X} \\
& =\left\|\int_{0}^{t}\left(\widetilde{P}_{h} \mathrm{e}^{(t-s) A_{h}} B_{h} Q_{h}-S(t-s) B\right) v(s) d s\right\|_{X} \\
& \leq C_{10} h^{s(1-\gamma) \theta} \int_{0}^{t} \frac{\|v(s)\|_{U}}{(t-s)^{\theta+(1-\theta) \gamma}} d s \\
& \leq \text { Cste } h^{s(1-\gamma) \theta}\|v\|_{L^{2}(0, T ; U)},
\end{aligned}
$$

and the conclusion follows.
First, using a dual version of (46), and using (21), and (13), one has

$$
\begin{aligned}
& \left\|\widetilde{P}_{h} \mathrm{e}^{t A_{h}} P_{h} y_{0}-S(t) y_{0}\right\|_{X} \\
& \leq\left\|\widetilde{P}_{h}\left(\mathrm{e}^{t A_{h}} P_{h} y_{0}-P_{h} S(t) y_{0}\right)\right\|_{X} \\
& +\left\|\left(\widetilde{P}_{h} P_{h}-I\right) S(t) y_{0}\right\|_{X} \\
& \leq C_{10} \frac{h^{s \theta}}{t^{\theta}}\left\|S(t) y_{0}\right\|_{X}+C_{4} h^{s}\left\|S(t) y_{0}\right\|_{X} \\
& \leq \operatorname{Cste} \frac{h^{s \theta}}{t^{\theta}}\left\|y_{0}\right\|_{X} \text {. }
\end{aligned}
$$

Second, for every $h \in\left(0, h_{1}\right)$, one gets, from a dual version of (45),

$$
\begin{aligned}
& \left\|\widetilde{P}_{h} L_{t h} u_{h}-L_{t} \widetilde{Q}_{h} u_{h}\right\|_{X} \\
& \leq \int_{0}^{t}\left\|\left(\widetilde{P}_{h} \mathrm{e}^{(t-s) A_{h}} B_{h}-S(t-s) B \widetilde{Q}_{h}\right) u_{h}(s) d s\right\|_{X} \\
& \leq \text { Cste } h^{s(1-\gamma) \theta}\left\|u_{h}\right\|_{L^{2}\left(0, T ; U_{h}\right)}^{2} \\
& \leq \text { Cste } h^{s(1-\gamma) \theta}\left\|y_{0}\right\|_{X}^{2} .
\end{aligned}
$$

Third, let us prove that $L_{t} \widetilde{Q}_{h} u_{h}$ converges strongly to $L_{t} u$ in $X$, for every $t \in[0, T]$. The operators $\widetilde{P}_{h} L_{t h} Q_{h}$ are of finite rank, and, from Lemma 4.2 , converge strongly to $L_{t}$. Hence, $L_{t}$ is a compact operator, for every $t \in[0, T]$.

Now, writing

$$
\begin{aligned}
& \widetilde{P}_{h} y_{h}(t)-y(t)=\left(\widetilde{P}_{h} \mathrm{e}^{t A_{h}} P_{h} y_{0}-S(t) y_{0}\right) \\
& +\left(\widetilde{P}_{h} L_{t h} u_{h}-L_{t} \widetilde{Q}_{h} u_{h}\right)+L_{t}\left(\widetilde{Q}_{h} u_{h}-u\right),
\end{aligned}
$$

it follows from (54), (55), from the weak convergence of $\widetilde{Q}_{h} u_{h}$ to $u$, and from the compactness of $L_{t}$, that, on the one hand, for every $t \in(0, T]$, the sequence $\left(\widetilde{P}_{h} y_{h}(t)\right)_{0<h<h_{1}}$ converges strongly in $X$ (up to subsequence) to $y(t)$, and, on the other hand, that the sequence $\left(\widetilde{P}_{h} y_{h}(\cdot)\right)_{0<h<h_{1}}$ converges strongly in $L^{2}(0, T ; X)$ (up to a subsequence) to $y(\cdot)$ on $[0, T]$,

In particular, since $y_{h}(T)=-h^{\beta} \varphi_{h} \rightarrow 0$ as $h$ tends to 0 , it follows that $y(T)=0$.

To prove the converse statement of Theorem 3.1 , it suffices to notice that, if the uniform inequality (35) holds, then, the sequence $\left(\widetilde{Q}_{h} u_{h}\right)$, where $u_{h}$ is defined by (37), and where $\varphi_{h}$ minimizes $J_{h}$, is bounded in $L^{2}$, and thus, up to a 
subsequence, converges to a control $u$. As previously, one proves that the trajectory $y(\cdot)$, solution of (38), associated with the control $u$, satisfies $y(T)=0$. Theorem 3.1 is proved.

\subsection{Proof of Proposition 3.2}

If the sequence $\left(\left\|\widetilde{P}_{h} \varphi_{h}\right\|_{X}\right)_{0<h<h_{1}}$ is bounded, then, up to a subsequence, it converges weakly to an element $\varphi \in X$. It follows from the estimate (45) that $u(t)=B^{*} S(T-t)^{*} \varphi$, for every $t \in[0, T]$. Moreover, $\widetilde{Q}_{h} u_{h}$ tends strongly to $u$ in $L^{2}(0, T ; U)$. Indeed, write, for every $t \in[0, T]$,

$$
\begin{aligned}
& \widetilde{Q}_{h} u_{h}(t)-u(t) \\
& =\left(\widetilde{Q}_{h} B_{h}^{*} \mathrm{e}^{(T-t) A_{h}^{*}}-B^{*} S(T-t)^{*} \widetilde{P}_{h}\right) \varphi_{h} \\
& \quad+B^{*} S(T-t)^{*}\left(\widetilde{P}_{h} \varphi_{h}-\varphi\right)
\end{aligned}
$$

Using (45), it is clear that the first term of the right-hand side of (56) tends to zero. For the second term, one first notes that, using the estimate (70) from the appendix, for every $t \in[0, T)$, the operator $B^{*} S(T-t)^{*}$ is compact, as a strong limit of finite rank operators. Since $\widetilde{P}_{h} \varphi_{h}-\varphi$ tends weakly to zero, it follows that the second term of the right-hand side of (56) tends to zero.

The control $u$ is such that $y(T)=0$, hence the vector $\varphi$ must be solution of $\nabla J(\varphi)=0$, where $J$ is defined by (11) (see Remark 2.1). Since $J$ is strictly convex, $\varphi$ is the minimum of $J$, that is, $u$ is the HUM control such that $y(T)=0$.

We next prove, by contradiction, that the sufficient condition provided in the statement of the proposition implies that the sequence $\left(\left\|\varphi_{h}\right\|_{X_{h}}\right)_{0<h<h_{1}}$ is bounded. If it is not bounded, then, up to subsequence, $\widetilde{P}_{h}\left(\varphi_{h} /\left\|\varphi_{h}\right\|_{X_{h}}\right)$ converges weakly to $\Phi \in X$, as $h$ tends to 0 . For every $t \in[T-\eta, T+\eta]$, the control system is exactly null controllable in time $t$, and thus, from (51), the sequence $\left\langle\mathrm{e}^{t A_{h}^{*}} \varphi_{h}, P_{h} y_{0}\right\rangle_{X_{h}}$ is bounded, uniformly for $h \in\left(0, h_{1}\right)$. Thus, passing to the limit, one gets $\left\langle\Phi, S(t) y_{0}\right\rangle_{X}=0$. This contradicts the fact that the trajectory $t \mapsto S(t) y_{0}, t \in[T-\eta, T+\eta]$, is not contained in a hyperplane of $X$.

\section{Numerical simulations for the heat equation with Neumann boundary con- trol}

In this section, we give an example of a situation where Theorem 3.1 applies, provide some numerical simulations, and comment on their practical implementation.

Let $d \geq 1$ be an integer, $c$ a real number, $\Omega$ an open bounded connected subset of $\mathbb{R}^{d}$, and $\Gamma=$ $\partial \Omega$. Let $\Gamma_{1}$ and $\Gamma_{2}$ be subsets of $\Gamma$ such that $\Gamma=$ $\Gamma_{1} \cup \Gamma_{2}$ and $\Gamma_{1} \cap \Gamma_{2}=\emptyset$. Consider the Neumann boundary control system

$$
\begin{aligned}
\frac{\partial y}{\partial t} & =\Delta y+c y & & \text { in }(0, T) \times \Omega, \\
y(0, \cdot) & =y_{0}(\cdot) & & \text { in } \Omega, \\
\frac{\partial y}{\partial n} & =u & & \text { on }[0, T] \times \Gamma_{1}, \\
\frac{\partial y}{\partial n} & =0 & & \text { on }[0, T] \times \Gamma_{2},
\end{aligned}
$$

where $y_{0} \in L^{2}(\Omega)$ and $u \in L^{2}\left(0, T ; L^{2}\left(\Gamma_{1}\right)\right)$.

Set $X=L^{2}(\Omega)$ and $U=L^{2}\left(\Gamma_{1}\right)$. It is well known (see $[17,22,23,24,25]$ ) that the control system (57) is exactly null controllable in $X$, with controls $u \in L^{2}(0, T ; U)$. It can be written in the form (38), where the selfadjoint operator $A: D(A) \rightarrow X$ is defined by $A y=\triangle y+c y$, on $D(A)=\left\{y \in H^{2}(\Omega) \mid \frac{\partial y}{\partial n}=0\right.$ on $\left.\Gamma\right\}$, and $B=-A N \in L\left(U, D\left(A^{*}\right)^{\prime}\right)$, where $N$ is the Neumann mapping, defined by

$$
N u=y \Leftrightarrow \begin{cases}A y=0 & \text { in } \Omega, \\ \frac{\partial y}{\partial n}=u & \text { on } \Gamma_{1}, \\ \frac{\partial y}{\partial n}=0 & \text { on } \Gamma_{2} .\end{cases}
$$

Note that $N: H^{s}\left(\Gamma_{1}\right) \longrightarrow H^{s+3 / 2}\left(\Gamma_{1}\right)$ is continuous, for every $s \in \mathbb{R}$. Here, we assume that $-c$ is not an eigenvalue of the Laplacian operator $\triangle$ on $D(A)$. The adjoint $B^{*} \in L\left(D\left(A^{*}\right), U\right)$ of $B$ is given by $B^{*} \psi=\psi_{\mid \Gamma_{1}}$, for every $\psi \in D\left(A^{*}\right)$. Morever, the degree of unboundedness of $B$ is $\gamma=1 / 4+\varepsilon$, for every $\varepsilon>0$. Hence, assumptions $\left(H_{1}\right)$ and $\left(H_{2}\right)$ are satisfied. Note that $D\left((-A)^{1 / 2}\right)=H^{1}(\Omega)$. 


\subsection{Finite element semi-discrete model}

We next introduce a semi-discretized model of the system (57), using finite elements of order one. Consider a family of simplex meshes $\left(\mathcal{T}_{n}\right)_{n \in \mathbb{N}}$ in $\mathbb{R}^{d}$, with $\mathcal{T}_{n}=\left(K_{k}\right)_{k \in\left\{1, \ldots, N_{n}\right\}}$, where, for every $k \in\left\{1, \ldots, N_{n}\right\}, K_{k}$ is an open $d$-simplex such that, for every $l \in\left\{1, \ldots, N_{n}\right\} \backslash\{k\}$, there holds $K_{k} \cap K_{l}=\emptyset$ and $\bigcup_{k=1}^{N_{n}} \bar{K}_{k}=\bar{\Omega}$. Let $\partial I_{n}$ be the set of indexes such that, for every $k \in \partial I_{n}$, there holds $\bar{K}_{k} \cap \Gamma_{1} \neq \emptyset$. Let $S_{n}$ be the number of distinct vertices $\left(p_{k}\right)_{k \in\left\{1, \ldots, S_{n}\right\}}$ of $\mathcal{T}_{n}$, and let $\partial S_{n}$ be the set of indexes of the distinct vertices of $\left(K_{k} \cap \Gamma_{1}\right)_{k \in \partial I_{n}}$. Let $h_{n}=\max _{k \in\left\{1, \ldots, N_{n}\right\}} \operatorname{diam}\left(K_{k}\right)$, where $\operatorname{diam}\left(K_{k}\right)$ denotes the diameter of $K_{k}$. Assume that $\left(h_{n}\right)_{n \in \mathbb{N}}$ is decreasing. For the sake of clarity, the index $n$ is replaced by $h$ in the following notations. Set

$X_{h}=\left\{y \in C^{0}(\Omega) \mid \forall k \in\left\{1, \ldots, N_{h}\right\}, y_{\mid K_{k}}\right.$ linear $\}$, $U_{h}=\left\{u \in C^{0}\left(\Gamma_{1}\right) \mid \forall k \in \partial I_{h}, y_{\mid K_{k} \cap \Gamma_{1}}\right.$ linear $\}$.

The spaces $X_{h}$ and $U_{h}$ are respectively generated by $\Phi_{h}=\left(\varphi_{k}\right)_{k \in\left\{1, \ldots, S_{h}\right\}}$ and $\Upsilon_{h}=\left(v_{k}\right)_{k \in \partial S_{h}}$, with

$$
\begin{aligned}
& \forall k \in\left\{1, \ldots, S_{h}\right\}, \varphi_{k}\left(p_{k}\right)=1, \\
& \forall l \in\left\{1, \ldots, S_{h}\right\} \backslash\{k\}, \varphi_{k}\left(p_{l}\right)=0, \\
& \forall k \in \partial S_{h}, v_{k}\left(p_{k}\right)=1, \\
& \forall l \in \partial S_{h} \backslash\{k\}, v_{k}\left(p_{l}\right)=0 .
\end{aligned}
$$

Note that $X_{h} \subset D\left((-A)^{1 / 2}\right)=H^{1}(\Omega)$ and $U_{h} \subset U$. Define $\widetilde{P}_{h}$ (resp., $\widetilde{Q}_{h}$ ), as the canonical injection from $X_{h}$ into $D\left((-A)^{1 / 2}\right)$ (resp., from $U_{h}$ into $\left.U\right)$. For all $x_{h}, y_{h} \in X_{h}$ and $u_{h}, v_{h} \in U_{h}$, set, according to $(25),\left\langle x_{h}, y_{h}\right\rangle_{X_{h}}=$ $\left\langle\widetilde{P}_{h} x_{h}, \widetilde{P}_{h} y_{h}\right\rangle_{X}$, and $\left\langle u_{h}, v_{h}\right\rangle_{U_{h}}=\left\langle\widetilde{Q}_{h} u_{h}, \widetilde{Q}_{h} v_{h}\right\rangle_{U}$. For every $y \in D\left((-A)^{1 / 2}\right)^{\prime}=H^{1}(\Omega)^{\prime}$ (with respect to the pivot space $\left.X=L^{2}(\Omega)\right)$, set $P_{h} y=$ $\left(M_{h}^{-1}\left\langle y, \widetilde{P}_{h} \Phi_{h}\right\rangle_{H^{1}(\Omega)^{\prime}, H^{1}(\Omega)}\right) . \Phi_{h}$, and, for every $u \in U$, set $Q_{h} u=\left(M_{\partial, h}^{-1}\left\langle u, \widetilde{Q}_{h} \Upsilon_{h}\right\rangle_{U}\right) . \Upsilon_{h}$, where $M_{h}=\left\langle\Phi_{h}, \Phi_{h}^{T}\right\rangle_{X_{h}}$, and $M_{\partial, h}=\left\langle\Upsilon_{h}, \Upsilon_{h}^{T}\right\rangle_{U_{h}}$, are mass matrices.

Assumptions $\left(H_{3.1}\right)$ and $\left(H_{3.3}\right)$ are obviously satisfied; the assumption $\left(H_{3.2}\right)$, with $s=2$, follows from the classical finite element theory (see
[5]), and the assumption $\left(H_{3.4}\right)$ follows from a standard approximation property (see [27]).

The variational version of (57) is the following. For $u \in C^{1}([0, T], U)$, one has to determine $y$ in $C^{1}([0, T], X)$ such that

$\left\langle y_{t}, w\right\rangle_{X}=-\langle\nabla u, \nabla w\rangle_{X}+c\langle y, w\rangle_{X}-\left\langle u, w_{\mid \Gamma_{1}}\right\rangle_{U}$, for every $w \in X$. We next derive a similar formulation in the approximating spaces $X_{h}$ and $U_{h}$, and, more precisely, in their respective representations in $\mathbb{R}^{S_{h}}$ and $\mathbb{R}^{\# \partial S_{h}}$. For $V \in C^{1}\left([0, T], \mathbb{R}^{\# \partial S_{h}}\right)$, one has to determine $Y \in$ $C^{1}\left([0, T], \mathbb{R}^{S_{h}}\right)$ such that

$$
\begin{aligned}
& \left\langle\dot{Y} . \Phi_{h}, W . \Phi_{h}\right\rangle_{X_{h}}=-\left\langle\nabla Y . \Phi_{h}, \nabla W . \Phi_{h}\right\rangle_{X_{h}} \\
& \quad+c\left\langle Y . \Phi_{h}, W . \Phi_{h}\right\rangle_{X_{h}}-\left\langle U . \Upsilon_{h}, W . \Phi_{h \mid \Gamma_{1}}\right\rangle_{U_{h}},
\end{aligned}
$$

for every $W \in \mathbb{R}^{S_{h}}$. Hence, the finite element semi-discretization model of (57) writes

$$
M_{h} \dot{Y}(t)=A_{h} Y(t)+B_{h} V(t), \quad Y(0)=Y_{0},
$$

where $Y_{0} \in \mathbb{R}^{S_{h}}, V(t) \in \mathbb{R}^{\# \partial S_{h}}, Y(t) \in \mathbb{R}^{S_{h}}$, and $A_{h}=-\left\langle\nabla \Phi_{h}, \nabla \Phi_{h}^{T}\right\rangle_{X_{h}}+c M_{h}, B_{h}=-\left\langle\Upsilon_{h}, \Phi_{h \mid \Gamma_{1}}^{T}\right\rangle_{U_{h}}$.

Remark 5.1. For implementation issues, this approximation model is not written in the abstract spaces $X_{h}$ and $U_{h}$, but rather in $\mathbb{R}^{S_{h}}$ and $\mathbb{R}^{\# \partial S_{h}}$. This does not alter the uniformity of the observability and admissibility inequality (35). Indeed, the mappings

$$
\begin{aligned}
\iota_{h}: X_{h} & \longrightarrow \mathbb{R}^{S_{h}} \\
y_{h} & \longmapsto M_{h}^{-1}\left\langle y_{h}, \Phi_{h}\right\rangle_{X}, \\
\iota_{\partial, h}: U_{h} & \longrightarrow \mathbb{R}^{\# \partial S_{h}} \\
u_{h} & \longmapsto M_{\partial, h}^{-1}\left\langle u_{h}, \Upsilon_{h}\right\rangle_{U},
\end{aligned}
$$

are isomorphisms, such that there exist $m>0$ and $M>0$ so that

$$
\begin{aligned}
& m\left\|y_{h}\right\|_{X_{h}} \leq\left\|\iota_{h}\left(y_{h}\right)\right\|_{\mathbb{R}^{S_{h}}} \leq M\left\|y_{h}\right\|_{X_{h}}, \\
& m\left\|u_{h}\right\|_{U_{h}} \leq\left\|\iota_{\partial, h}\left(u_{h}\right)\right\|_{\mathbb{R}^{\# \partial S_{h}}} \leq M\left\|u_{h}\right\|_{U_{h}},
\end{aligned}
$$

for every $y_{h} \in X_{h}$, and every $u_{h} \in U_{h}$. This follows from a standard property of the spectrum of mass matrices (see [5]).

The uniform analyticity assumption $\left(H_{4.1}\right)$ follows from [3]. The assumption $\left(H_{4.2}\right)$ is satisfied with $s=2$ (see $[5,12,27])$. Hence, Theorem 3.1 applies, with $\beta=0.45$ for instance. 


\subsection{Numerical simulations}

The minimization procedure described in Theorem 3.1 has been implemented for $d=1$ and $d=2$, using a standard gradient type method, that has the advantage not to require the computation of the gradient of the functional. Indeed, this computation is expensive, since the gradient is related to the Gramian matrix. In the following numerical simulations, provided using Matlab, we choose $c=1$. Then, the operator $A$ has a positive eigenvalue, and the uncontrolled system (57) (i.e., $u \equiv 0)$ is unstable.

\subsubsection{The one-dimensional heat equation}

Set $\Omega=(0,1), \Gamma_{1}=\{1\}, \Gamma_{2}=\{0\}, c=1$, and $T=1$. With the previous notations, consider the subdivision of $\Omega, p_{k}=(k-1) h$, for $k \in\left\{1, \ldots, S_{h}\right\}$ and $h=1 /\left(S_{h}-1\right)$. Numerical simulations are carried out with a time discretization step equal to 0.001 , with the data of Table 1 . The numerical results are provided on Table 2 . The notation $y_{h}^{u}(T)$ of this table stands for the extremity at time $T$ of the solution of (57), for $u_{h} \equiv 0$.

The convergence of the method is slow. For an exact time integration, the final state $y_{h}(T)$ is equal to $-h^{\beta} \varphi_{h}$. This is however in accordance with the estimates (41) of Theorem 3.1. Indeed, it follows from these estimates that $y_{h}(T)$ converges very slowly to zero (here, $\beta / 2=0.225$ ). These results illustrate the difficulty in using the HUM method to compute controls. In our case, to divide $\left\|y_{h}(T)\right\|_{X_{h}}$ by 10 , one has to divide $h$ by 30000 . On the other part, three days of computations are required, on a bi-processor (Xeon $2.40 \mathrm{GHz}, 512$ Mo RAM), to compute controls for $h=10^{-3}$. Nevertheless, the convergence of the gradient procedure is fast and does not seem to depend on the size of the space discretization (6 to 8 iterations, in our numerical simulations). Hence, optimizing the computation of $J_{h}$ may improve the performance of the algorithm and decrease computation times.

\subsubsection{The two-dimensional heat equation}

Let $\Omega$ be the unit disk of $\mathbb{R}^{2}$, let $c=1$, and $T=1$. Numerical simulations are carried out with a time discretization step equal to 0.001 , with the data of Table 3. The numerical results are provided on Table 4. The bench disk_1 gives slightly better results than the bench disk_2, due to the fact that the control is located on one part of the boundary only. The simulations provided here involve 55 and 104 cells (see Figure 1). As previously, the times of computations are very long, and, to divide $\left\|y_{h}(T)\right\|_{X_{h}}$ by 10 , one has to divide the cell diameters by 30000 , that is, one has to multiply the number of cells by $9.10^{8}$; this is clearly not feasible on a standard machine, within reasonable time, and indicates the limits of the method.

\section{Conclusion and open prob- lems}

We have shown that, under standard assumptions on the discretization process, for an exactly null controllable linear control system, if the semigroup of the approximating system is uniformly analytic, and if the degree of unboundedness of the control operator is lower than $1 / 2$, then the semidiscrete approximation models are uniformly controllable, in a certain sense. This property is weaker than the uniform exact null controllability (that does not hold in general). A minimization procedure was provided to build the approximation controls, and moreover a uniform observability and admissibility type inequality was proved, that implies a uniform conditioning of the minimization problem, and thus, the number of iterations of the gradient method is uniformly bounded. This was implemented in the case of the one- and two-dimensional heat equation with Neumann boundary control.

No rates of convergence were given for the convergence of controls of the semidiscrete models, and this is an open problem.

The condition on the degree of unboundedness $\gamma$ of the control operator $B$ is very stringent, and an interesting open problem is to investigate whether the results of this article still hold whenever $\gamma \geq 1 / 2$. Note that, if $\gamma<1 / 2$, then $B$ is automatically admissible; this does not hold necessarily whenever $\gamma \geq 1 / 2$, and may cause some technical difficulties. However, there are many im- 
portant and relevant problems for which $\gamma \geq 1 / 2$, that are not covered by the results of this paper, such as, for instance, the heat equation with Dirichlet boundary control. Note that, in this case, the finite difference semidiscrete models are uniformly exactly null controllable in the one dimensional case (see [16]), but lose this property in larger dimensions in general (see [33]). There are some important physical examples in which the semigroup is analytic and $\gamma \geq 1 / 2$, such as thermo-elastic plates coupling a parabolic dynamics with an Euler-Bernoulli dynamics (see [10] and [12, Chap. 3.11]).

Another open question is to remove the assumption of uniform analyticity of the discretized semigroup. The properties of analyticity of the semigroup have been used in an essential way in the proof of the results (see Remark 3.4). The situation is widely open for hyperbolic equations. Note that, for the 1-D wave equation, a result of uniform controllability was proved when using a mixed finite element discretization process (see [4]); the extension to higher dimensions is not clear (see [33]). However, a general result, stating uniform stabilization properties, was derived in [20] for general hyperbolic systems, and it would be interesting to try to adapt the techniques of proof used in this paper to the problem of exact controllability. As mentioned in Remark 3.4, it is possible to derive a result similar to Theorem 3.1 , combining exact controllability with the convergence of the numerical scheme; however this argument does not lead a priori to a uniform observability type inequality, and thus, to a uniform conditioning. Preliminary numerical simulations in some hyperbolic situations indeed indicate that the number of iterations of the gradient method is not bounded, as $h$ tends to zero.

Acknowledgement. We thank E. Zuazua for many remarks and stimulating discussions.

\section{Appendix: proof of Lemma}

\section{1}

We first prove (43). Using $\left(H_{1}\right)$, there holds, for every $t>0$ (see [19]), $S(t)^{*}=$ $\frac{1}{2 i \pi} \int_{\partial \Delta_{\delta}} \mathrm{e}^{\lambda t} R\left(\lambda, A^{*}\right) d \lambda$, and, similarly, $\mathrm{e}^{t A_{h}^{*}}=$ $\frac{1}{2 i \pi} \int_{\partial \Delta_{\delta}} \mathrm{e}^{\lambda t} R\left(\lambda, A_{h}^{*}\right) d \lambda$. It follows that

$$
\begin{aligned}
& \mathrm{e}^{t A_{h}^{*}} P_{h}-P_{h} S(t)^{*} \\
& =\frac{1}{2 i \pi} \int_{\partial \Delta_{\delta}} \mathrm{e}^{\lambda t}\left(R\left(\lambda, A^{*}\right) P_{h}-P_{h} R\left(\lambda, A_{h}^{*}\right)\right) d \lambda .
\end{aligned}
$$

From the resolvent identity $R\left(\beta_{1}, A\right)=$ $R\left(\beta_{2}, A\right)+\left(\beta_{2}-\beta_{1}\right) R\left(\beta_{1}, A\right) R\left(\beta_{2}, A\right)$, one gets $R\left(\lambda, A_{h}^{*}\right) P_{h}=R\left(\omega, A_{h}^{*}\right) P_{h}+(\omega-$ 入) $R\left(\lambda, A_{h}^{*}\right) R\left(\omega, A_{h}^{*}\right) P_{h}$, and $P_{h} R\left(\lambda, A^{*}\right)=$ $P_{h} R\left(\omega, A^{*}\right)+(\omega-\lambda) P_{h} R\left(\lambda, A^{*}\right) R\left(\omega, A^{*}\right)$. It follows that

$$
\begin{aligned}
& \left.\left(R\left(\lambda, A_{h}^{*}\right) P_{h}-P_{h} R\left(\lambda, A^{*}\right)\right)\left(I-(\lambda-\omega) \hat{A}^{*-1}\right)\right) \\
& \left.=\left(I+(\omega-\lambda) R\left(\lambda, A_{h}^{*}\right)\right)\left(\hat{A}_{h}^{*-1}\right) P_{h}-P_{h} \hat{A}^{*-1}\right) .
\end{aligned}
$$

Note that $I-(\lambda-\omega) \hat{A}^{*-1}=\left(\hat{A}^{*}-(\lambda-\omega) I\right) \hat{A}^{*-1}$, and thus, $\left(I-(\lambda-\omega) \hat{A}^{*-1}\right)^{-1}=-\hat{A} R(\lambda-\omega, \hat{A})$. Using (17), one gets

$$
\left\|\left(I-(\lambda-\omega) \hat{A}^{*-1}\right)^{-1}\right\|_{L(X)} \leq C_{2},
$$

for every $\lambda \in \Delta_{\delta}$. On the other hand, from (33),

$$
\left\|(\omega-\lambda) R\left(\lambda, A_{h}^{*}\right)\right\|_{L\left(X_{h}\right)} \leq C_{8} .
$$

We deduce from (60), (61), and (62), that there exists a constant $C_{15}$ such that

$$
\begin{aligned}
& \left\|R\left(\lambda, A_{h}^{*}\right) P_{h}-P_{h} R\left(\lambda, A^{*}\right)\right\|_{L\left(X, X_{h}\right)} \\
& \quad \leq C_{15}\left\|\hat{A}_{h}^{*-1} P_{h}-P_{h} \hat{A}^{-1}\right\|_{L\left(X, X_{h}\right)},
\end{aligned}
$$

for every $\lambda \in \Delta_{\delta}$. The estimate (43) follows from (59), (63), and from (34). We next prove (44). For every $\psi \in D\left(A^{*}\right)$, one has

$$
\begin{aligned}
& \left\|\widetilde{Q}_{h} B_{h}^{*} \mathrm{e}^{t A_{h}^{*}} P_{h} \psi-B^{*} \widetilde{P}_{h} P_{h} S(t)^{*} \psi\right\|_{U} \\
& \quad \leq\left\|\widetilde{Q}_{h} B_{h}^{*} \mathrm{e}^{t A_{h}^{*}} P_{h} \psi\right\|_{U}+\left\|B^{*} \widetilde{P}_{h} P_{h} S(t)^{*} \psi\right\|_{U} .
\end{aligned}
$$

We estimate each term of the right-hand side of (64). From (31), $B_{h}^{*}=Q_{h} B^{*} \widetilde{P}_{h}$, and thus, using (26), (27), (30), and (32), one gets

$$
\begin{aligned}
\left\|\widetilde{Q}_{h} B_{h}^{*} \mathrm{e}^{t A_{h}^{*}} P_{h} \psi\right\|_{U} & \leq C_{5}\left\|B^{*} \widetilde{P}_{h} \mathrm{e}^{t A_{h}^{*}} P_{h} \psi\right\|_{U} \\
& \leq C_{5} C_{6} h^{-\gamma s}\left\|\mathrm{e}^{t A_{h}^{*}} P_{h} \psi\right\|_{X_{h}} \\
& \leq C_{5}^{2} C_{6} C_{7} h^{-\gamma s} \mathrm{e}^{\omega t}\|\psi\|_{X}
\end{aligned}
$$


On the other hand, from (30), (27), and (13),

$$
\begin{aligned}
\left\|B^{*} \widetilde{P}_{h} P_{h} S(t)^{*} \psi\right\|_{U} & \leq C_{6} h^{-\gamma s}\left\|P_{h} S(t)^{*} \psi\right\|_{X_{h}} \\
& \leq C_{5} C_{6} h^{-\gamma s}\left\|S(t)^{*} \psi\right\|_{X} \\
& \leq C_{1} C_{5} C_{6} h^{-\gamma s} \mathrm{e}^{\omega t}\|\psi\|_{X} .
\end{aligned}
$$

Hence, using (64), (65), and (66), there exists $C_{16}>0$ such that

$$
\begin{gathered}
\left\|\widetilde{Q}_{h} B_{h}^{*} \mathrm{e}^{t A_{h}^{*}} P_{h} \psi-B^{*} \widetilde{P}_{h} P_{h} S(t)^{*} \psi\right\|_{U} \\
\leq C_{16} h^{-\gamma s}\|\psi\|_{X},
\end{gathered}
$$

for every $\psi \in D\left(A^{*}\right)$, every $t \in[0, T]$, and every $h \in\left(0, h_{0}\right)$. Let us get another estimate of this term. Using successively (27), (30), (19), (24), (43), (22), and (13), one gets

$$
\begin{aligned}
& \left\|\widetilde{Q}_{h} B_{h}^{*} \mathrm{e}^{t A_{h}^{*}} P_{h} \psi-B^{*} \widetilde{P}_{h} P_{h} S(t)^{*} \psi\right\|_{U} \\
& =\left\|\widetilde{Q}_{h} Q_{h} B^{*} \widetilde{P}_{h} \mathrm{e}^{t A_{h}^{*}} P_{h} \psi-B^{*} \widetilde{P}_{h} P_{h} S(t)^{*} \psi\right\|_{U} \\
& \leq\left\|\widetilde{Q}_{h} Q_{h} B^{*} \widetilde{P}_{h}\left(\mathrm{e}^{t A_{h}^{*}} P_{h} \psi-P_{h} S(t)^{*} \psi\right)\right\|_{U} \\
& +\left\|\widetilde{Q}_{h} Q_{h} B^{*}\left(\widetilde{P}_{h} P_{h}-I\right) S(t)^{*} \psi\right\|_{U} \\
& +\left\|\left(\widetilde{Q}_{h} Q_{h}-I\right) B^{*} S(t)^{*} \psi\right\|_{U} \\
& \quad+\left\|B^{*}\left(I-\widetilde{P}_{h} P_{h}\right) S(t)^{*} \psi\right\|_{U} \\
& \leq C_{5} C_{6} h^{-\gamma s}\left\|\mathrm{e}^{t A_{h}^{*}} P_{h} \psi-P_{h} S(t)^{*} \psi\right\|_{X_{h}} \\
& \quad+C_{5} C_{3}\left\|(-\hat{A})^{\gamma}\left(\widetilde{P}_{h} P_{h}-I\right) S(t)^{*} \psi\right\|_{X} \\
& \quad+C_{4} h^{s(1-\gamma)}\left\|A^{*} S(t)^{*} \psi\right\|_{X} \\
& \quad+C_{3}\left\|(-\hat{A})^{\gamma}\left(\widetilde{P}_{h} P_{h}-I\right) S(t)^{*} \psi\right\|_{X} \\
& \leq C_{5} C_{6} C_{10} \frac{h^{s(1-\gamma)}}{t}\|\psi\|_{X} \\
& \quad+\left(C_{3}\left(C_{5}+1\right)+1\right) C_{4} h^{s(1-\gamma)}\left\|A^{*} S(t)^{*} \psi\right\|_{X} \\
& \leq C_{17} \frac{h^{s(1-\gamma)}}{t}\|\psi\|_{X},
\end{aligned}
$$

for every $\psi \in D\left(A^{*}\right)$, every $t \in[0, T]$, and every $h \in\left(0, h_{0}\right)$, where $C_{17}>0$. Then, raising (67) to the power $1-\gamma,(68)$ to the power $\gamma$, and multiplying both resulting estimates, we obtain $\left\|\widetilde{Q}_{h} B_{h}^{*} \mathrm{e}^{t A_{h}^{*}} P_{h} \psi-B^{*} \widetilde{P}_{h} P_{h} S(t)^{*} \psi\right\|_{U} \leq \frac{C_{18}}{t^{\gamma}}\|\psi\|_{X}$, and hence,

$$
\begin{aligned}
& \left\|\widetilde{Q}_{h} B_{h}^{*} \mathrm{e}^{t A_{h}^{*}} P_{h} \psi\right\|_{U} \\
& \quad \leq \frac{C_{18}}{t^{\gamma}}\|\psi\|_{X}+\left\|B^{*} \widetilde{P}_{h} P_{h} S(t)^{*} \psi\right\|_{U} .
\end{aligned}
$$

From (19), (28), and (14), there holds

$$
\begin{aligned}
& \left\|B^{*} \widetilde{P}_{h} P_{h} S(t)^{*} \psi\right\|_{U} \\
& \leq\left\|B^{*}\left(\widetilde{P}_{h} P_{h}-I\right) S(t)^{*} \psi\right\|_{U}+\left\|B^{*} S(t)^{*} \psi\right\|_{U} \\
& \leq C_{3}\left(\left\|(-\hat{A})^{\gamma}\left(\widetilde{P}_{h} P_{h}-I\right) S(t)^{*} \psi\right\|_{X}\right. \\
& \left.+\left\|(-\hat{A})^{\gamma} S(t)^{*} \psi\right\|_{X}\right) \\
& \leq C_{3}\left(C_{5}+1\right)\left\|(-\hat{A})^{\gamma} S(t)^{*} \psi\right\|_{X} \\
& \leq C_{3}\left(C_{5}+1\right) C_{1} \frac{\mathrm{e}^{\omega t}}{t^{\gamma}}\|\psi\|_{X}
\end{aligned}
$$

and thus, using (69), the estimate (44) follows by setting $\psi=\widetilde{P}_{h} \psi_{h}$ and using (20).

Finally, we prove (45). On the one hand, reasoning as above for obtaining (68), we get

$$
\left\|\widetilde{Q}_{h} B_{h}^{*} \mathrm{e}^{t A_{h}^{*}} P_{h} \psi-B^{*} S(t)^{*} \psi\right\|_{U} \leq C_{19} \frac{h^{s(1-\gamma)}}{t}\|\psi\|_{X},
$$

for every $\psi \in D\left(A^{*}\right)$, every $t \in[0, T]$, and every $h \in\left(0, h_{0}\right)$, where $C_{19}$ is a positive constant. On the other hand, from (44), (19), and (14), setting $\psi=\widetilde{P}_{h} \psi_{h}$,

$$
\begin{aligned}
& \left\|\widetilde{Q}_{h} B_{h}^{*} \mathrm{e}^{t A_{h}^{*}} P_{h} \psi-B^{*} S(t)^{*} \psi\right\|_{U} \\
& \leq\left\|\widetilde{Q}_{h} B_{h}^{*} \mathrm{e}^{t A_{h}^{*}} P_{h} \psi\right\|_{U}+\left\|B^{*} S(t)^{*} \psi\right\|_{U} \\
& \leq \frac{C_{10}}{t^{\gamma}}\left\|\psi_{h}\right\|_{X_{h}}+C_{3}\left\|\left(-\hat{A}^{*}\right)^{\gamma} S(t)^{*} \psi\right\|_{X} \\
& \leq \frac{C_{20}}{t^{\gamma}}\left\|\psi_{h}\right\|_{X_{h}},
\end{aligned}
$$

where $C_{20}>0$. Raising (70) to the power $\theta,(71)$ to the power $1-\theta$, and multiplying both resulting estimates, we obtain (45). Lemma 4.1 is proved.

\section{References}

[1] H.T. Banks, K. Ito, Approximation in LQR problems for infinite dimensional systems with unbounded input operators, J. Math. Systems Estim. Control 7, 1 (1997).

[2] H.T. Banks, K. Kunisch, The linear regulator problem for parabolic systems, SIAM J. Control Optim. 22,5 (1984) 684-698.

[3] J. Bramble, A. Shatz, V. Thomee, L. Wahlbin, Some convergence estimates for semidiscrete Galerkin type approximations for parabolic equations, SIAM J. Num. Anal. 14 (1977) 218-241. 
[4] C. Castro, S. Micu, Boundary controllability of a linear semi-discrete $1-\mathrm{D}$ wave equation derived from a mixed finite element method, to appear, Numer. Math.

[5] A. Ern, J.L. Guermond, Theory and practice of finite elements, Applied Mathematical Series, 159, Springer-Verlag, New-York, 2004.

[6] J.S. Gibson, The Riccati integral equations for optimal control problems on Hilbert spaces, SIAM J. Control Optim. 17 (1979) 537-565.

[7] R. Glowinski, J.-L. Lions, Exact and approximate controllability of distributed parameter systems, Acta Numerica (1996) 159-333.

[8] J.A. Infante, E. Zuazua, Boundary observability for the space semi-discretizations of the 1-D wave equation, M2AN Math. Model. Numer. Anal. 33, 2 (1999) 407-438.

[9] F. Kappel, D. Salamon, An approximation theorem for the algebraic Riccati equation, SIAM J. Control Optim. 28, 5 (1990) 1136-1147.

[10] J. Lagnese, Boundary stabilization of thin plates, SIAM (1989).

[11] I. Lasiecka, Convergence estimates for semidiscrete approximations of nonselfadjoint parabolic equations, SIAM J. Num. Anal. 21, 5 (1977) 894-908.

[12] I. Lasiecka, R. Triggiani, Control theory for partial differential equations: continuous and approximation theories. I. Abstract parabolic systems, Encyclopedia of Mathematics and its Applications, 74, Cambridge University Press, Cambridge, 2000.

[13] L. Leon, E. Zuazua, Boundary controllability of the finite-difference space semi-discretizations of the beam equation, ESAIM Control Optim. Calc. Var. 8 (2002) 827-862.

[14] J.-L. Lions, Exact controllability, stabilization and perturbations for distributed systems, SIAM Rev. 30 (1988) 1-68.

[15] Z. Liu, S. Zheng, Semigroups associated with dissipative systems, Research Notes Math. 398, 1999.

[16] A. Lopez, E. Zuazua, Some new results related to the null controllability of the 1-d heat equation, Sém. EDP, Ecole Polytechnique, VIII, 1998, 1-22.

[17] V.J. Mizel, T.I. Seidman, Observation and prediction for the heat equation, J. Math. Anal. Appl. 28 (1969) 303-312.

[18] M. Negreanu, E. Zuazua, Uniform boundary controllability of a discrete 1-D wave equation, Systems Control Lett. 48, 3-4 (2003) 261-279.

[19] A. Pazy, Semigroups of linear operators and applications to partial differential equations, Applied Math. Sci. 44, Springer-Verlag, 1983.

[20] K. Ramdani, T. Takahashi, M. Tucsnak, Uniformly exponentially stable approximations for a class of second order evolution equations, Preprint Univ. Nancy, 2004.

[21] R. Rebarber, G. Weiss, Necessary conditions for exact controllability with a finite-dimensional input space, Syst. Control Lett. 40, 3 (2000) 217-227.
[22] D.L. Russell, A unified boundary controllability theory for hyperbolic and parabolic partial differential equations, Studies in Appl. Math. 52 (1973) 189-211.

[23] D.L. Russell, Controllability and stabilizability theory for linear partial differential equations: recent progress and open questions, SIAM Rev. 20, 4 (1978) 639-739.

[24] T.I. Seidman, Observation and prediction for the heat equation, III, J. Diff. Eq. 20, 1 (1976) 18-27.

[25] T.I. Seidman, Observation and prediction for the heat equation, IV, Patch observability and controllability, SIAM J. Cont. Opt. 15, 3 (1977) 412-427.

[26] L.R. Tcheugoué Tébou, E. Zuazua, Uniform exponential long time decay for the space semidiscretization of a locally damped wave equation via an artificial numerical viscosity, Numer. Math. 95, 3 (2003) 563-598.

[27] V. Thomée, Galerkin finite element methods for parabolic problems, Lecture Notes in Mathematics, Springer-Verlag, 1984.

[28] G. Weiss, Admissible observation operators for linear semigroups, Israel J. Math. 65, 1 (1989) 17-43.

[29] G. Weiss, Admissibility of unbounded control operators, SIAM J. Cont. Opt. 27, 3 (1989) 527-545.

[30] E. Zuazua, Boundary observability for the finitedifference space semi-discretizations of the 2-D wave equation in the square, J. Math. Pures Appl. 9, 78, 5 (1999) 523-563.

[31] E. Zuazua, Controllability of partial differential equations and its semi-discrete approximations, Discrete Contin. Dyn. Syst. 8, 2 (2002) 469-513.

[32] E. Zuazua, Optimal and approximate control of finite-difference approximation schemes for the 1-D wave equation, Rendiconti di Matematica VIII, 24, II (2004) 201-237.

[33] E. Zuazua, Propagation, Observation, Control and Numerical Approximation of Waves approximated by finite difference method, SIAM Review 47, 2 (2005) 197-243.

[34] E. Zuazua, Controllability and observability of partial differential equations: some results and open problems, in HandBbook of Differential Equations: Evolutionary Differential Equations, C. Dafermos and E. Feireisl eds., Elsevier Science, to appear.

[35] E. Zuazua, Control and numerical approximation of the wave and the heat equations, Proceedings Internat. Congress Math. (2006), to appear. 


\begin{tabular}{|c|c|c|c|}
\hline name & $S_{h}$ & $h$ & $y_{0}$ \\
\hline \hline 1D_10 & 11 & $10^{-1}$ & $y_{0}(x)=x$ \\
1D_100 & 101 & $10^{-2}$ & $y_{0}(x)=x$ \\
1D_1000 & 1001 & $10^{-3}$ & $y_{0}(x)=x$ \\
sin1D_10 & 11 & $10^{-1}$ & $y_{0}(x)=\sin ^{2}(\pi x)$ \\
sin1D_100 & 101 & $10^{-2}$ & $y_{0}(x)=\sin ^{2}(\pi x)$ \\
sin1D_1000 & 1001 & $10^{-3}$ & $y_{0}(x)=\sin ^{2}(\pi x)$ \\
\hline
\end{tabular}

Table 1: Data for the one-dimensional heat equation

\begin{tabular}{|c|c|c|c|c|c|}
\hline name & $\left\|\psi_{h}\right\|_{X}$ & $h^{\beta}$ & $\left\|h^{\beta} \psi_{h}+y_{h}(T)\right\|_{X}$ & $\left\|y_{h}(T)\right\|_{X_{h}}$ & $\left\|y_{h}^{u}(T)\right\|_{X_{h}}$ \\
\hline \hline 1D_10 & 0.41380476 & 0.3720411 & 0.0030567 & 0.1567023 & 1.3577812 \\
1D_100 & 0.53185422 & 0.1264632 & 0.0036999 & 0.0700055 & 1.3577812 \\
1D_1000 & 0.70536645 & 0.0446885 & 0.0039907 & 0.0339719 & 1.3577812 \\
sin1D_10 & 0.41383134 & 0.3720411 & 0.0030884 & 0.1567131 & 1.3577812 \\
sin1D_100 & 0.53222526 & 0.1264632 & 0.0040701 & 0.0700721 & 1.3577812 \\
sin1D_1000 & 0.70565021 & 0.0446885 & 0.0039909 & 0.0339841 & 1.3577812 \\
\hline
\end{tabular}

Table 2: Numerical results for the one-dimensional equation

\begin{tabular}{|c|c|c|c|c|}
\hline name & $S_{h}$ & $y_{0}$ & $\Gamma_{1}$ & $\Gamma_{2}$ \\
\hline \hline disk_1 & 55 & $y_{0}(x, y)=x+y$ & $\Gamma$ & $\emptyset$ \\
& 104 & $y_{0}(x, y)=x+y$ & $\Gamma$ & $\emptyset$ \\
disk_2 & 55 & $y_{0}(x, y)=x+y$ & $\{(x, y) \in \Gamma \mid x \geq 0$ and $y \geq 0\}$ & $\Gamma \backslash \Gamma_{1}$ \\
& 104 & $y_{0}(x, y)=x+y$ & $\{(x, y) \in \Gamma \mid x \geq 0$ and $y \geq 0\}$ & $\Gamma \backslash \Gamma_{1}$ \\
\hline
\end{tabular}

Table 3: Data for the two-dimensional equation

\begin{tabular}{|c|c|c|c|c|c|c|}
\hline name & $S_{h}$ & $\left\|\psi_{h}\right\|_{X}$ & $h^{\beta}$ & $\left\|h^{\beta} \psi_{h}+y_{h}(T)\right\|_{X}$ & $\left\|y_{h}(T)\right\|_{X_{h}}$ & $\left\|y_{h}^{u}(T)\right\|_{X_{h}}$ \\
\hline \hline disk_1 & 55 & 0.1587292 & 0.6142423 & 0.0174738 & 0.0803068 & 0.1035126 \\
& 104 & 0.2309178 & 0.4511246 & 0.0195391 & 0.0849494 & 0.1085287 \\
disk_2 & 55 & 0.1649749 & 0.6142423 & 0.0116253 & 0.0962398 & 0.1035126 \\
& 104 & 0.1751393 & 0.4511246 & 0.0106206 & 0.1025904 & 0.1086115 \\
\hline
\end{tabular}

Table 4: Numerical results for the two-dimensional equation
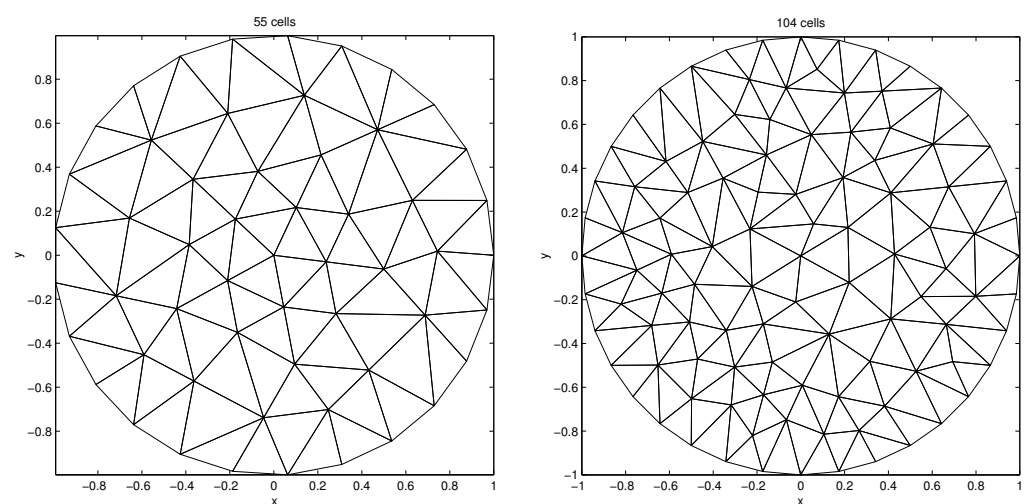

Figure 1: Meshes used for disk benches. 